\title{
E3, Rat Strain
}

National Cancer Institute

\section{Source}

National Cancer Institute. E3, Rat Strain. NCI Thesaurus. Code C14400.

Has platelet storage disease associated with the red-eyed yellow locus, as described by Raymond and Dodds (1975) for the FH strain. 